\title{
Analysis of an epidemic model for transmitted diseases in a group of adults and an extension to two age classes
}

\author{
Meltem Gölgeli*1 (D), Fatihcan M. $\operatorname{Atay}^{2}$ (D) \\ ${ }^{1}$ Department of Mathematics, TOBB University of Economics and Technology, 06510 Ankara, Turkey \\ ${ }^{2}$ Department of Mathematics, Bilkent University, 06800 Ankara, Turkey
}

\begin{abstract}
Infectious diseases are a serious problem for public health and spark the interest in interdisciplinary studies. In this paper, we present two mathematical models describing a possible scenario for infectious diseases. The first model considers the dynamics of the disease among adults and emphasizes the role of carriers in the SIR model and the second model assumes that the disease is transmitted to children by adults. We state the equilibria for each model and study the local stability of the equilibria. Furthermore, we perform simulations using a parameter set that explains the spread of a specific infectious disease (meningococcal disease) and interpret the possible cases of transmission via simulations.
\end{abstract}

Mathematics Subject Classification (2010). 92B05, 37N25, 62P10

Keywords. SIR model, epidemic threshold, local stability, age structure

\section{Introduction}

Although infectious diseases have a long past in human history, we still have to fight against many of them, e.g. the common flu, SARS, measles, malaria, tuberculosis, AIDS, meningitis etc. It is possible to reduce the mortality due to infectious diseases by medical care and vaccinations. However, bacterial and viral agents evolve simultaneously and mutate against the medical treatments. Mathematical modeling of infectious diseases allows us to understand the dynamics of transmission of a disease within the population. Subsequently, the aim is to predict the possible behavior of the disease, which might enable controlling a possible epidemic through treatment, vaccination or isolation policies.

It is generally accepted that the first mathematical model in epidemiology is published in 1790 by Daniel Bernoulli, who studied the effect of cowpox inoculation on the spread of smallpox by using nonlinear ordinary differential equations. In more recent times, Kermack and McKendrick [18] produced a generalized epidemic model in 1927, which categorises a population as a candidate of an infection in three distinct compartments: susceptible, infected, and recovered. Their deterministic approach for a uniformly distributed population is still widely studied and has a key role in the new modeling aspects concerning factors like age, immigration-migration, vaccination, and the effect of several therapy methods or

\footnotetext{
*Corresponding Author.

Email addresses: mgolgeli@etu.edu.tr (Meltem Gölgeli), f.atay@bilkent.edu.tr (Fatihcan M. Atay)

Received: 09.07.2018; Accepted: 14.05.2019
} 
quarantine even for a non-uniformly distributed population. The mathematical model of Kermack-McKendrick is given by

$$
\begin{aligned}
\frac{d S}{d t} & =-\kappa S I, \\
\frac{d I}{d t} & =\kappa S I-\lambda I, \\
\frac{d R}{d t} & =\lambda I,
\end{aligned}
$$

where $S(t), R(t), I(t)$, respectively, represent the number of susceptible, infective and recovered individuals at time $t$, and $\kappa$ and $\lambda$ are the transmission rate and the recovery rate, respectively. According to the hypothesis of conservation of the population, we have $S(t)+I(t)+R(t)=N$, where $N$ is the constant population size [16,24]. This types of mathematical models are known as classical SIR (susceptible, infective, and recovered) models and yield important results for epidemics. Assuming that in the beginning of an epidemic the number of susceptibles, infectives and recovereds are given by $S(0)=S_{0}>0$, $I(0)=I_{0}>0$ and $R(0)=0$. Then, we define the basic reproduction number $\mathfrak{R}_{\mathrm{o}}=\kappa S_{0} / \lambda$. This is a threshold value, in the sense that epidemic spreads if $\mathfrak{R}_{0}>1$ and dies out if $\mathfrak{R}_{\mathrm{o}}<1$, which is an important outcome of an epidemic model, saying when it is possible to control the spread of the disease [16,24]. In general, analyzing the nonlinear system (1.1) is not simple since explicit solutions are usually impossible to obtain and small changes in the system may have a strong affect on the complexity of the dynamics. However, there are some approaches to find exact analytical solution in parametric form for such epidemic models [13]. Additionally, behavior related to particular equilibrium solutions can be studied by the well-known tools of stability analysis. Local and global stability of SIR models are extensively studied in the literature [12,19, 23, 28].

The basic model (1.1) can be enhanced in several ways to address more realistic situations. For example, real populations are not homogeneous, age being one of the most important factors of heterogeneity. Individuals in different age groups may have different infection tendencies [5,25]. Indeed, childhood diseases (measles, chicken pox, rubella etc.) spread mainly through contacts between children of similar ages. Furthermore, young people have an active social life and children meet many others in their age group at school every day. A higher contact rate within the community increases the disease transmission rate.

In this paper, we discuss the stability analysis of an SIR epidemic model with and without an age structure. We assume two age classes, namely adults and kids, and ignore the spatial heterogeneity of age classes. We construct the SIR model in the form of an ordinary differential equation (ODE) system, and examine the disease-free and endemic equilibria for both with and without age structure.

\section{Mathematical model without age structure and its stability analysis}

The schematic diagram in Figure 1 presents the essence of our first model. We categorize the population into four compartments: $S_{A}(t)$ represents susceptible adults who are candidates for contracting the disease, $I_{A V}(t)$ denotes infected adults who do not show any symptoms of the disease although they continue to transmit it, $I_{A N}(t)$ is infected adults who do show the symptoms of the disease, and $R_{A}(t)$ denotes adult individuals who have recovered. In point of fact, infected people in the compartment $I_{A V}(t)$ are vaccinated adults who transmit the disease to the unvaccinated adults in the compartment $I_{A N}(t)$. We consider a fixed homogeneous population, where the age of adults has no effect on the probability of being infected. Dividing the compartment of infected individuals into the subclasses is needed for some modeling aspects. Indeed, in many infectious diseases there 


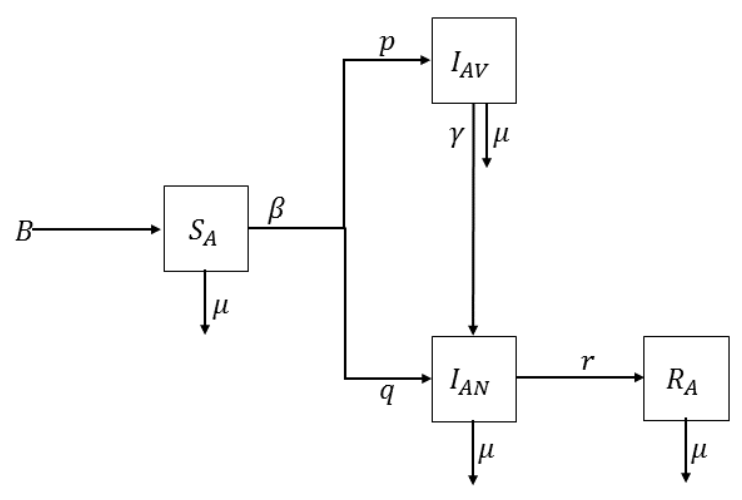

Figure 1. Diagram describing the first model given by equation system (2.1)

are individuals who 'carry' the virus or bacteria that cause the disease without showing any symptoms of the disease. For example, SIR models explaining AIDS often suggest two classes of infected individuals: the first class of infectives represents HIV carriers without a symptom of AIDS, whereas the second class consists of infectives who exhibit the symptoms of the disease [2]. Centers for Disease Control and Prevention, Atlanta, USA, reported that travelers may be at increased risk for meningococcal disease, caused by $N$. meningitidis, if they travel to certain places, and recommended meningococcal vaccines for people who are traveling to these high-risk areas [8]. Although meningococcal vaccination influences carriage prevalence in vaccinated individuals, it could not prevent the transmission of disease [6]. So, even if the vaccinated travellers do not show the symptoms of meningococcal disease, they may transmit it. As a second example, B. pertussis may prevail among vaccinated people who do not display symptoms, and an asymptomatic transmission is also possible [3]. Biologically, the vaccinated adults could be also carriers and transmit a disease to the other adults, as in meningococcal disease or whooping cough (Bordetella pertussis).

Thus, our first modelling scenario considers the case that a group of vaccinated adult travellers are infected abroad and they carry the bacteria to their home. We assume that the bacterial transmission is only possible among adults. The serogroup of bacteria transmitted by vaccinated adults could be totally different although the infected unvaccinated individuals show similiar symptoms [9,30]. A mathematical model for infectious diseases that include the effects of carriers on the transmission dynamics is proposed by [17]. We use a similar idea to construct the mathematical model explaining the transmission dynamics between vaccinated and unvaccinated adults expressed as

$$
\begin{aligned}
\frac{d S_{A}}{d t} & =B-\mu S_{A}-\beta S_{A}\left(p I_{A V}+q I_{A N}\right) \\
\frac{d I_{A V}}{d t} & =\beta p S_{A} I_{A V}-\mu I_{A V}-\gamma I_{A V} \\
\frac{d I_{A N}}{d t} & =\beta q S_{A} I_{A N}-\mu I_{A N}-r I_{A N}+\gamma I_{A V} \\
\frac{d R_{A}}{d t} & =r I_{A N}-\mu R_{A},
\end{aligned}
$$

with the initial conditions $S_{A}(0) \geq 0, I_{A V}(t) \geq 0, I_{A N}(t) \geq 0$ and $R_{A}(0) \geq 0$. Since, the total population density is assumed to be constant,

$$
S_{A}(t)+I_{A V}(t)+I_{A N}(t)+R_{A}(t)=1 .
$$

Hence, we have $S_{A}^{\prime}(t)+I_{A V}^{\prime}(t)+I_{A N}^{\prime}(t)+R_{A}^{\prime}(t)=0$. 
Table 1. Parameters of the model (2.1)

\begin{tabular}{clc}
\hline Parameters & Units & Description \\
\hline$\beta$ & $(1 /$ year $)$ & transmission rate \\
$p$ & none & probability of transmitting the infection for the compartment $I_{A V}$ \\
$q$ & none & probability of transmitting the infection for the compartment $I_{A N}$ \\
$\gamma$ & $(1 /$ year $)$ & rate of showing symptoms $I_{A V}$ to $I_{A N}$ \\
$\mu$ & $(1 /$ year $)$ & natural death rate \\
$r$ & $(1 /$ year $)$ & recovery rate \\
$B$ & $(1 /$ year $)$ & birth rate \\
\hline
\end{tabular}

The parameters of the model (2.1) are summarized in Table 1.

Contrary to the model in [17], all of the individuals in the compartment $I_{A V}$ are vaccinated carriers and we do not need to define an extra compartment for vaccinated individuals. Further, the transmission by interaction between $S_{A}$ and $I_{A V}$ results in the class $I_{A V}$ with the transmission rate $\beta p$ and the transmission by interaction between $S$ and $I_{A N}$ results in the class $I_{A N}$ with the transmission rate $\beta q$. Thus, the constant $\beta$ could be extent a time dependent parameter $\beta(t)$ while analysing a traveller case as mentioned in [8]. Moreover, here it is important to discuss the parameters $p$ and $q$ controlled by vaccination policy. In this sense, the stability analysis of the equilibria is different in our case.

If there is no infection in the population, we have $I_{A V}=I_{A N}=0$, which is known as the disease-free state. Since there are no infected individuals, we do not have any recovered individuals, i.e. $R_{A}=0$. While seeking the disease-free equilibria, it is sufficient to find the equilibrium for the non-zero class $S_{A}(0)$. Thus, the disease-free equilibrium has the form $E_{d f}=\left(S_{A}^{*}, 0,0,0\right)$, where we obtain $S_{A}^{*}=\frac{B}{\mu}$ using the system

$$
\begin{aligned}
B-\mu S_{A}-\beta S_{A}\left(p I_{A V}+q I_{A N}\right) & =0 \\
\beta p S_{A} I_{A V}-\mu I_{A V}-\gamma I_{A V} & =0 \\
\beta q S_{A} I_{A N}-\mu I_{A N}-r I_{A N}+\gamma I_{A V} & =0 \\
r I_{A N}-\mu R_{A} & =0 .
\end{aligned}
$$

Similarly, we assume to be at the endemic state if there is any infected person in the population, i.e., $I_{A V}>0, I_{A N}>0, R=0$. At this state the endemic equilibrium reads $E_{d}=\left(S_{A}^{*}, I_{A V}^{*}, I_{A N}^{*}, R_{A}^{*}\right)$, where

$$
\begin{aligned}
S_{A}^{*} & =\frac{\mu+\gamma}{\beta p}, \\
I_{A V}^{*} & =-\frac{1}{\gamma p}(q(\mu+\gamma)-p(\mu+r)) I_{A N}^{*}, \\
I_{A N}^{*} & =\gamma\left(\frac{B p}{\mu+\gamma}-\frac{\mu}{\beta}\right)(-q \mu+p(\mu+r))^{-1}, \\
R_{A}^{*} & =\frac{r}{\mu} I_{A N}^{*},
\end{aligned}
$$

using (2.2). On the basis of (2.3), the dynamics for $R$ can be derived from the first three equations. In this sense, we reduce the system and analyze the stability of $E_{d}=$ $\left(S_{A}^{*}, I_{A V}^{*}, I_{A N}^{*}\right)$. 
We evaluate the Jacobian matrix of the system (2.2) for analyzing the local stability of the disease-free and endemic equilibrium points:

$$
J=\left[\begin{array}{ccc}
-\mu-\beta\left(p I_{A V}^{*}+q I_{A N}^{*}\right) & -\beta p S_{A}^{*} & -\beta q S_{A}^{*} \\
\beta p I_{A V}^{*} & \left(\beta p S_{A}^{*}-\mu-\gamma\right) & 0 \\
\beta q I_{A N}^{*} & \gamma & \left(\beta q S_{A}^{*}-\mu-r\right)
\end{array}\right] .
$$

We first consider the disease-free equilibrium $E_{\mathrm{df}}=\left(S_{A}^{*}, 0,0\right)$, where the Jacobian (2.4) takes the form

which has the eigenvalues

$$
J_{\mathrm{df}}=\left[\begin{array}{ccc}
-\mu & -\beta p \frac{B}{\mu} & -\beta q \frac{B}{\mu} \\
0 & \left(\beta p \frac{B}{\mu}-\mu-\gamma\right) & 0 \\
0 & \gamma & \left(\beta q \frac{B}{\mu}-\mu-r\right)
\end{array}\right],
$$

$$
\lambda_{1}=-\mu, \quad \lambda_{2}=\left(B \frac{\beta p}{\mu}-\mu-\gamma\right), \quad \lambda_{3}=\left(B \frac{\beta q}{\mu}-\mu-r\right) .
$$

The disease-free equilibrium is locally asymptotically stable if the real parts of all eigenvalues are negative. It is clear that $\lambda_{1}<0$, since $\mu>0$ because of the biological assumptions. Stipulating that $\lambda_{2}$ and $\lambda_{3}$ also be negative yields the conditions for the stability of the disease-free equilibrium as

$$
\beta p \frac{B}{\mu}<\mu+\gamma \quad \text { and } \quad \beta(1-p) \frac{B}{\mu}<\mu+r .
$$

We have determined the endemic equilibrium point $E_{d}=\left(S_{A}^{*}, I_{A V}^{*}, I_{A N}^{*}\right)$ given by equations (2.3). For simplicity, we rewrite $E_{d}=\left(S_{A}^{*}, W I_{A N}^{*}, I_{A N}^{*}\right)$ with $W=-\frac{1}{\gamma p}(q(\mu+\gamma)-p(\mu+r))$. Substituting the endemic equilibrium state $E_{d}=\left(S_{A}^{*}, W I_{A N}^{*}, I_{A N}^{*}\right)$ into the Jacobian (2.4) gives

$$
J_{d}=\left[\begin{array}{ccc}
-\mu-\beta(p W+q) I_{A N}^{*} & -(\mu+\gamma) & -\frac{q}{p}(\mu+\gamma) \\
\beta p W I_{A N}^{*} & 0 & 0 \\
\beta q I_{A N}^{*} & \gamma & (\mu+\gamma) \frac{q}{p}-\mu-r
\end{array}\right] .
$$

Now the characteristic polynomial has the form $\lambda^{3}+a_{2} \lambda^{2}+a_{1} \lambda+a_{0}=0$, where

$$
\begin{aligned}
& a_{2}=2 \mu+r-(\mu+\gamma) \frac{q}{p}+\beta(p W+q) I_{A N}^{*} \\
& a_{1}=\left(-\mu-\beta(p W+q) I_{A N}^{*}\right)\left((\mu+\gamma) \frac{q}{p}-\mu-r\right)+(\mu+\gamma)\left(\beta p W I_{A N}^{*}\right)+\frac{q}{p}(\mu+\gamma) \beta q I_{A N}^{*} \\
& a_{0}=-\beta p W I_{A N}^{*}(\mu+\gamma)\left(\left(\frac{q}{p}(\mu+\gamma)-\mu-r\right)+\frac{q}{p} \gamma\right) .
\end{aligned}
$$

According to the Routh-Hurwitz stability criterion, the roots are in $\mathbb{C}_{-}$if and only if $a_{2}>0$ and $a_{1} a_{2}>a_{0}>0$, in which case the equilibrium $E_{d}$ is locally asymptotically stable.

Under the assumption $0<S_{A}^{*} \leq \frac{B}{\mu}$ we obtain the basic reproduction number $\mathfrak{R}_{\mathrm{o}}=$ $\frac{B \beta p}{(\mu+\gamma) \mu}$ using the Jacobian matrix and next generation method [11].

\section{Simulations for the model without age structure}

We have carried out simulations for a population of 100 individuals assuming that the birth and death rates are equal to each other, which ensures a constant population size by assuming not to have a significant change in the population size in a few months. Turkish Statistical Institute (TUIK) has reported the life expactancy in Turkey to be around 
around 78 in year 2016. Thus we assume $B=\mu=0.0128$. Since we aim to understand how fast the vaccinated adults transmit a disease to other adults, we mainly concentrate on the parameter $\beta$ and $\gamma$. The parameter $p$ represents the probability of vaccination that effects the number of the individuals in the classes $I_{A V}$ and $I_{A N}$. In that sense we assume a moderate vaccination policy and take the value of $p$ hypothetically equal to 0.4. As a suitable example to the model (2.2) we point out the meningococcal disease. World Health Organisation suggest people vaccination for meningococcal disease if they are travelling to sub-Saharan Africa. Additionally, pilgrims travelling to the Kingdom of Saudi Arabia for the Hajj or Umra are required to show a proof vaccination in order to obtain a visa. However, the meningococcal disease carriage is reported in Turkish hajj pilgrims [9]. The transmission rate $\beta$ of meningitis varies depending on environmental and geographical conditions [22]. Ozsurekci claimed in her doctoral thesis that the bacterial meningitis prevalence in Turkey is 3.5 among 100000 individuals per year [27]. Thus, we assume an initial rate for transmission $\beta=0.035$ and we vary the infection rate $\beta$ to manipulate an epidemic or disease-free state of the population. We assume a fixed rate of showing symptoms $\gamma=0.1$, as calculated for the lowest rate at which carriers become ill in African meningitis belt [15]. Although the fatality rate in patients with meningitis is between $10 \%$ and $26 \%$, some patients may survive with neurologic sequelae [22]. We define 'recovery' in our research basically as 'being alive', which is strongly dependent on the age of the patient. However, the treatment duration is between 5 and 30 days, where 5 to 7 days will be sufficient for an uncomplicated case of meningococcal disease [14]. Since an individual needs approximately 5 to 7 days to recover from disease, we assume the recovery rate $r=0.6$.

The stability conditions and the nature of stability can be found in Table 2 for the disease-free state, and in Table 3 for the epidemic state.

Table 2. Stability of the disease-free state

\begin{tabular}{|c|c|c|c|c|c|c|c|c|c|c|}
\hline$B=\mu$ & $\beta$ & $p$ & $q$ & $\gamma$ & $r$ & $\lambda_{1}$ & $\lambda_{2}$ & $\lambda_{3}$ & $\mathfrak{R}_{\mathcal{o}}$ & Stability \\
\hline 0.0128 & $\mathbf{0 . 0 3 5}$ & 0.4 & 0.6 & 0.1 & 0.6 & -0.0128 & -0.1114 & -0.6107 & 0.1241 & Stable \\
0.0128 & $\mathbf{0 . 2 8 2 1}$ & 0.4 & 0.6 & 0.1 & 0.6 & -0.0128 & -0.0000002 & -0.4430 & 1 & Neutrally stable \\
0.0128 & $\mathbf{0 . 3}$ & 0.4 & 0.6 & 0.1 & 0.6 & -0.0128 & 0.0072 & -0.4328 & 1.0636 & Unstable \\
\hline
\end{tabular}

Table 3. Stability of the endemic state

\begin{tabular}{|c|c|c|c|c|c|c|c|c|c|}
\hline$B=\mu$ & $\beta$ & $p$ & $q$ & $\gamma$ & $r$ & $a_{2}$ & $a_{1} a_{2}>a_{0}>0$ & $\mathfrak{R}_{\mathfrak{o}}$ & Stability \\
\hline 0.0128 & $\mathbf{0 . 0 3 5}$ & 0.4 & 0.6 & 0.1 & 0.6 & $-0.00005<0$ & $a_{1} a_{2}>a_{0}$ & 0.1241 & Unstable \\
0.0128 & $\mathbf{0 . 2 8 2 1}$ & 4.2 & 0.6 & 0.1 & 0.6 & $0.4564>0$ & $a_{1} a_{2}>a_{0}>0$ & 1 & Neutrally stable \\
0.0128 & $\mathbf{0 . 3}$ & 0.4 & 0.6 & 0.1 & 0.6 & $0.4572>0$ & $0.028>0.0004$ & 1.0636 & Stable \\
\hline
\end{tabular}

Figure 2 shows the variation of $S_{A}, I_{A V}$, and $I_{A N}$ as a function of time for diseasefree and endemic cases. The presented model may apply to many infectious diseases that have two sub-classes of the compartment for infectious individuals. One of these classes is generally defined as a compartment for carriers. For example, diseases like HIV infection, Hepatitis B infection or bacterial meningitis have such a modeling structure. Particularly, we concentrate on the bacterial meningitis case and use parameters related to bacterial meningitis. Since we do not have a data set for the epidemic scenario, we may roughly estimate the transmission behaviour of the disease. The simulations support the prediction that as long as $\mathfrak{R}_{0}$ remains low (i.e. $\mathfrak{R}_{0}<1$ ), the disease may disappear, whereas if $\mathfrak{R}_{0}$ is high (i.e. $\mathfrak{R}_{0}>1$ ), the disease tends to spread. This result can be applied to any model of infectious disease that consists of individuals who are infected without showing any symptom and people who are infected and showing the symptoms. 


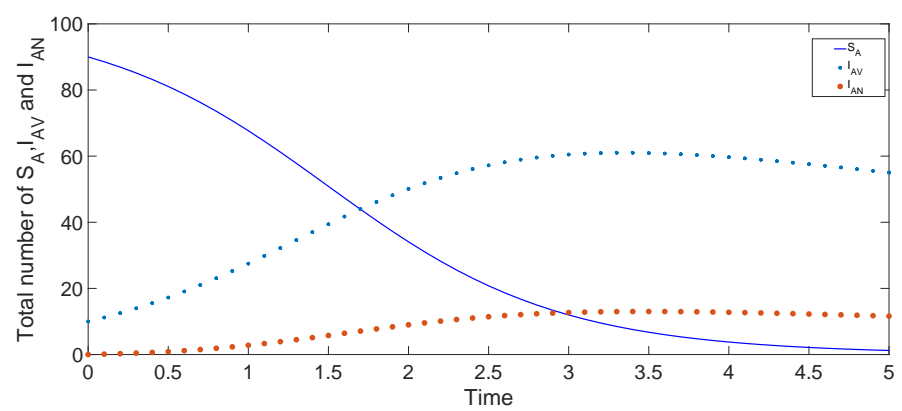

(a) $\beta=0.035$

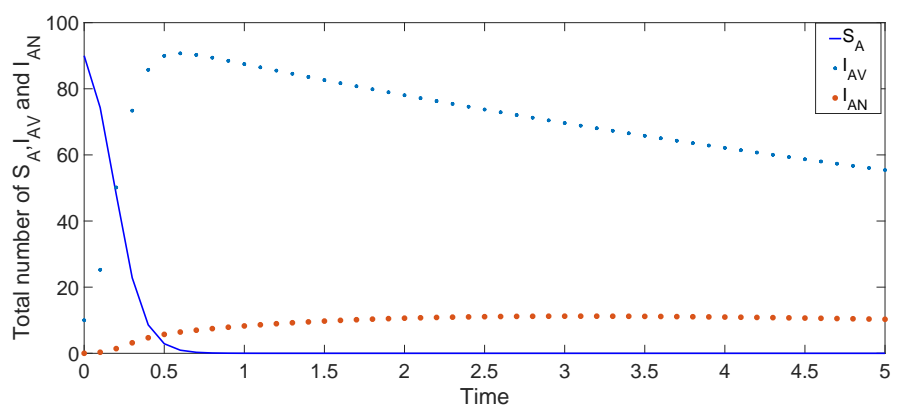

(b) $\beta=0.2821$

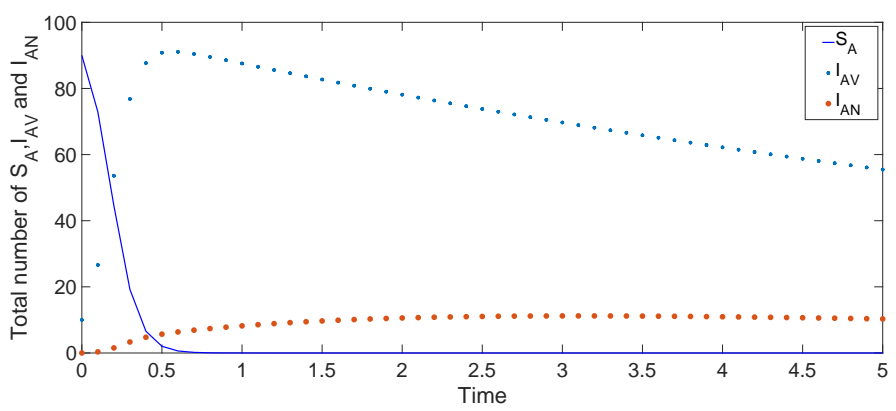

(c) $\beta=0.3$

Figure 2. The variation of the $S_{A}, I_{A V}$ and $I_{A N}$ classes for various values of the infectious rate $\beta$. (a) $\beta=0.035$, (b) $\beta=0.2821$, (c) $\beta=0.3$

Eventually, we construct a mathematical model which represents the transmission dynamics between vaccinated and unvaccinated adults. The main idea is that the vaccination policy of countries do generally target the adults who are visiting risky regions. However, the vaccinated adults could carry the infection to their children, grandchildren etc. [9]. Thus, we aim to extend the model (2.1) for two age classes, assuming the vaccinated travellers transmit a disease to the children in their environment.

\section{Mathematical model of two age classes and its stability analysis}

Generally, the risk of transmission of an infection depends on the age and immunity status of susceptible individuals. For example, [20] describes adults as virus reservoirs for children for the measles virus transmission, [10] reports that about half of all cases of bacterial meningitis in USA occur in children between 0-18 years of age, and [4] emphasizes that adult travelers with rotavirus shed 10-100 times less rotavirus than do pediatric 
patients. The transmission processes have different results for different diseases. Nevertheless, almost every age-structured deterministic modeling approach follows the realistic age-structured measles model of [29]. We depict our case diagrammatically in Figure 3.

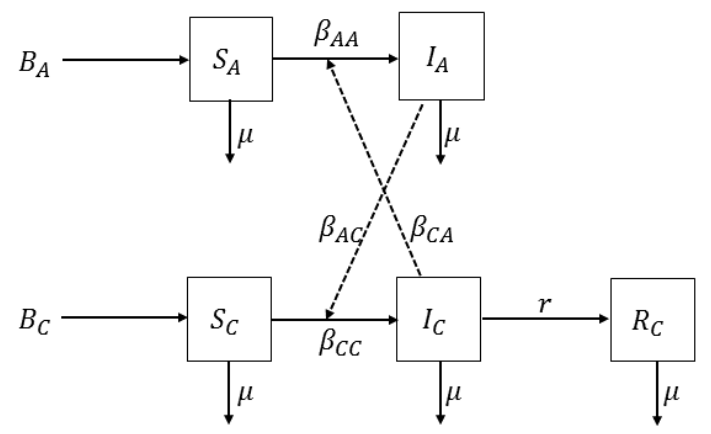

Figure 3. Schematic description of the model (4.1).

Since we have analyzed the effect of adult carriers in our previous model, we assume to have only a single class of adult carriers which are able to transmit the disease to children. In that sense, we only assume to have vaccinated adults in the model (4.1), because we have roughly estimated that even in endemic case the vaccinated adults are the main reservoir of the infection. Namely, we ignore that the unvaccinated adults (who are not travellers) could also be infected and transmit the infection to the children. All adults have gained immunity, e.g. through vaccination, and after a trip to abroad they might carry the bacteria to the children in the family. Since they do not show any symptoms of the disease, the recovery process for adults is neglected. The population is divided into two age classes as in [7]. Although people of any age can develop bacterial meningitis, children are at increased risk for bacterial meningitis [26]. In reality, the prevalence of the disease is different in age subgroups of children. We do not define the age threshold between adult and child classes clearly, just because we do not analyse any other age subgroup of children. We use similar notation as in the previous model and let $S_{C}, I_{C}$, and $R_{C}$ represent the susceptible, infectious, and recovered compartments, respectively. The total population density is $N=N_{A}+N_{C}=1$, where the indexes $A$ and $C$ represent the age classes 'adult' and 'children'. We explain the parameters of model (4.1) in Table 4. Contrary to the model for adults (2.1), we assume that all adults are vaccinated but they are able to carry the infection without showing any symptoms. That is why a recovery compartment for adults is omitted. All of the susceptible children are unvaccinated; so they can get the infection, transmit it and recover from the disease. As in the previous model (2.1), we assume that all surviving children are recovered, although some of them may survive with neurologic sequelae [22]. The age-structured model is then described by the system of differential equations

$$
\begin{aligned}
\frac{d S_{A}}{d t} & =B_{A}-\left(\beta_{A A} I_{A}+\beta_{C A} I_{C}\right) S_{A}-\mu S_{A} \\
\frac{d S_{C}}{d t} & =B_{C}-\left(\beta_{C C} I_{C}+\beta_{A C} I_{A}\right) S_{C}-\mu S_{C} \\
\frac{d I_{A}}{d t} & =\left(\beta_{A A} I_{A}+\beta_{C A} I_{C}\right) S_{A}-\mu I_{A} \\
\frac{d I_{C}}{d t} & =\left(\beta_{C C} I_{C}+\beta_{A C} I_{A}\right) S_{C}-\mu I_{C}-r I_{C} \\
\frac{d R_{C}}{d t} & =r I_{C}-\mu R_{C} .
\end{aligned}
$$


Defining the total population by $N=S_{A}+S_{C}+I_{A}+I_{C}+R_{C}$ means that $R_{C}$ can be obtained uniquely; thus, we omit the equation for $d R_{C} / d t$ and reduce the system of equations (4.1).

Table 4. Parameters of the model (4.1)

\begin{tabular}{ccc}
\hline Parameters & Units & Description \\
\hline$\beta_{A A}, \beta_{A C}$ & $1 /$ year & transmission rates between adults and from adults to children, respectively \\
$\beta_{C C}, \beta_{C A}$ & 1 year & transmission rates between children and from children to adults, respectively \\
$\mu$ & $1 /$ year & natural death rate \\
$r$ & $1 /$ year & recovery rate for children \\
$B_{C}$ & $1 /$ year & attendance rate of vaccinated adult travellers \\
$B_{C}$ & $1 /$ year & birth rate for children \\
\hline
\end{tabular}

The parameters of the model (4.1) are summarized in Table 4.

The disease-free state corresponds to $I_{A}=I_{C}=0$. Since there are no infected children at the beginning, there are also no recovered children, i.e. $R_{C}=0$. Thus the disease-free equilibrium has the form of $E_{d f}=\left(S_{A}^{*}, S_{C}^{*}, 0,0\right)$, where we obtain $S_{A}^{*}=\frac{B_{A}}{\mu}$ and $S_{C}^{*}=\frac{B_{C}}{\mu}$ using the model equations (4.1).

The Jacobian matrix of the system (4.1) at the equilibrium points is given by

$J=\left[\begin{array}{cccc}-\left(\beta_{A A} I_{A}^{*}+\beta_{C A} I_{C}^{*}\right)-\mu & 0 & -\beta_{A A} S_{A}^{*} & -\beta_{C A} S_{A}^{*} \\ 0 & -\left(\beta_{C C} I_{C}+\beta_{A C} I_{A}\right)-\mu & -\beta_{A C} S_{C}^{*} & -\beta_{C C} S_{C}^{*} \\ \beta_{A A} I_{A}^{*}+\beta_{C A} I_{C}^{*} & 0 & \beta_{A A} S_{A}^{*}-\mu & \beta_{C A} S_{A}^{*} \\ 0 & \beta_{C C} I_{C}+\beta_{A C} I_{A} & \beta_{A C} S_{C}^{*} & \beta_{C C} S_{C}^{*}-\mu-r\end{array}\right]$.

At the disease-free equilibrium $E_{d f}=\left(S_{A}^{*}, S_{C}^{*}, 0,0\right)$, the Jacobian matrix takes the form

$$
J_{\mathrm{df}}=\left[\begin{array}{cccc}
-\mu & 0 & -\beta_{A A} \frac{B_{A}}{\mu} & -\beta_{C A} \frac{B_{A}}{\mu} \\
0 & -\mu & -\beta_{A C} \frac{B_{C}}{\mu} & -\beta_{C C} \frac{B_{C}}{\mu} \\
0 & 0 & \beta_{A A} \frac{B_{A}}{\mu}-\mu & \beta_{C A} \frac{B_{A}}{\mu} \\
0 & 0 & \beta_{A C} \frac{B_{C}}{\mu} & \beta_{C C} \frac{B_{C}}{\mu}-\mu-r
\end{array}\right] .
$$

The eigenvalues are

where

$$
\lambda_{1,2}=-\mu, \quad \lambda_{3,4}=\frac{-b \pm \sqrt{b^{2}-4 c}}{2},
$$

$$
b=-\left(\beta_{A A} \frac{B_{A}}{\mu}+\beta_{C C} \frac{B_{C}}{\mu}-2 \mu-r\right)
$$

and

$$
c=\left(\beta_{A A} \frac{B_{A}}{\mu}-\mu\right)\left(\beta_{C C} \frac{B_{C}}{\mu}-\mu-r\right)-\left(\beta_{C A} \beta_{A C} \frac{B_{A} B_{C}}{\mu^{2}}\right) .
$$

The necessary and sufficient condition for the local asymptotic stability of equilibria is that the real parts of all eigenvalues be negative. Because of the biological feasibility, $\lambda_{1,2}=-\mu$ is always negative. If $b^{2}-4 c \geq 0$, we have real roots $\lambda_{3}$ and $\lambda_{4}$ so that $\lambda_{3}+\lambda_{4}=-b<0$ and $\lambda_{3} \lambda_{4}=\frac{b^{2}-\left(b^{2}-4 c\right)}{4}=c>0$. If $b^{2}-4 c<0$, we have complex conjugate roots $\lambda_{3}$ and $\lambda_{4}$ where $\lambda_{3} \lambda_{4}=\left(\frac{-b-i \sqrt{b^{2}-4 c}}{2}\right)\left(\frac{-b+i \sqrt{b^{2}-4 c}}{2}\right)=b^{2}-2 c>0$. Thus, the local stability condition appears as $2 c<b^{2}<4 c$.

Since the analytical treatment of the dynamical model (4.1) is difficult, we will make the simplifying assumption that $\beta_{C A}=0$, that is, the children are not able to transmit the disease to the adults. We state that all adults in our model are 'carriers' who do not show any symptoms of the disease and do not need to recover from the disease. This additional 
assumption does not damage the main modeling idea but allows us to reduce the system (4.1). Recall that $R_{C}$ can be obtained from (4.1) separately; hence we omit it. We thus obtain

$$
\begin{aligned}
\frac{d S_{A}}{d t} & =B_{A}-\beta_{A A} I_{A} S_{A}-\mu S_{A} \\
\frac{d S_{C}}{d t} & =B_{C}-\left(\beta_{C C} I_{C}+\beta_{A C} I_{A}\right) S_{C}-\mu S_{C} \\
\frac{d I_{A}}{d t} & =\beta_{A A} I_{A} S_{A}-\mu I_{A} \\
\frac{d I_{C}}{d t} & =\left(\beta_{C C} I_{C}+\beta_{A C} I_{A}\right) S_{C}-\mu I_{C}-r I_{C} .
\end{aligned}
$$

The endemic equilibrium has the form $E_{d}=\left(S_{A}^{*}, S_{C}^{*}, I_{A}^{*}, I_{C}^{*}\right)$, where $S_{A}^{*}=\frac{\mu}{\beta_{A A}}$ and $I_{A}^{*}=\frac{1}{\mu}\left(B_{A}-\frac{\mu^{2}}{\beta_{A A}}\right)$, and we find the dependent system

$$
\begin{gathered}
S_{C}^{*}=\frac{B_{C}}{\beta_{C C} I_{C}^{*}+\frac{\beta_{A C}}{\mu}\left(B_{A}-\frac{\mu^{2}}{\beta_{A A}}\right)+\mu}, \\
I_{C}^{*}=\left(\beta_{C C} I_{C}^{*}+\frac{\beta_{A C}}{\mu}\left(B_{A}-\frac{\mu^{2}}{\beta_{A A}}\right)\right)\left(\frac{B_{C}}{\beta_{C C} I_{C}^{*}+\frac{\beta_{A C}}{\mu}\left(B_{A}-\frac{\mu^{2}}{\beta_{A A}}\right)+\mu}\right) \frac{1}{\mu+r} .
\end{gathered}
$$

Some algebra yields

$$
I_{C}^{*}=\frac{-b \pm \sqrt{\Delta}}{2 a}
$$

where

$$
\begin{aligned}
a & =B_{C} \beta_{C C}^{2}-(\mu+r) \beta_{C C}, \\
b & =\left(B_{C}\left(2 \beta_{C C} \frac{\beta_{A C}}{\mu}\left(B_{A}-\frac{\mu^{2}}{\beta_{A A}}\right)+\beta_{C C} \mu\right)\right), \\
c & =B_{C}\left(\left(\frac{\beta_{A C}}{\mu}\left(B_{A}-\frac{\mu^{2}}{\beta_{A A}}\right)\right)^{2}+\beta_{A C}\left(B_{A}-\frac{\mu^{2}}{\beta_{A A}}\right)\right), \text { and } \\
\Delta & =b^{2}-4 a c .
\end{aligned}
$$

Thus, we obtain the equilibria $E_{d 1}=\left(S_{A}^{*}, S_{C 1}^{*}, I_{A}^{*}, I_{C 1}^{*}\right)$ and $E_{d 2}=\left(S_{A}^{*}, S_{C 2}^{*}, I_{A}^{*}, I_{C 2}^{*}\right)$. All parameters are positive as they have to be biologically meaningful. We also simplify the associated Jacobian matrix assuming $\beta_{C A}=0$ :

$$
\begin{aligned}
J_{\mathrm{d}} & =\left[\begin{array}{cccc}
-\beta_{A A} I_{A}^{*}-\mu & 0 & -\beta_{A A} S_{A}^{*} & 0 \\
0 & -\left(\beta_{C C} I_{C}+\beta_{A C} I_{A}\right)-\mu & -\beta_{A C} S_{C}^{*} & -\beta_{C C} S_{C}^{*} \\
\beta_{A A} I_{A}^{*} & 0 & \beta_{A A} S_{A}^{*}-\mu & 0 \\
0 & \beta_{C C} I_{C}+\beta_{A C} I_{A} & \beta_{A C} S_{C}^{*} & \beta_{C C} S_{C}^{*}-\mu-r
\end{array}\right] \\
& =\left[\begin{array}{cccc}
\bar{A} & 0 & \bar{B} & 0 \\
0 & \bar{C} & \bar{D} & \bar{E} \\
\bar{F} & 0 & \bar{G} & 0 \\
0 & \bar{H} & \bar{I} & \bar{J}
\end{array}\right]
\end{aligned}
$$


For local stability we consider the eigenvalues of $J_{\mathrm{d}}$, which are roots of the characteristic polynomial $\lambda^{4}+b_{3} \lambda^{3}+b_{2} \lambda^{2}+b_{1} \lambda+b_{0}=0$, where

$$
\begin{aligned}
& b_{3}=-(\bar{A}+\bar{G}+\bar{C}+\bar{J}), \\
& b_{2}=(\bar{A} \bar{G}-\bar{B} \bar{F}+\bar{C} \bar{J}-\bar{E} \bar{H}+(\bar{C}+\bar{J})(\bar{A}+\bar{G})), \\
& b_{1}=\bar{A} \bar{H} \bar{E}+\bar{G} \bar{H} \bar{E}-(\bar{C}+\bar{J})(\bar{A} \bar{G}-\bar{B} \bar{F})-(\bar{A}+\bar{G}) \bar{C} \bar{J}, \\
& b_{0}=\bar{C} \bar{J}(\bar{A} \bar{G}-\bar{B} \bar{F})+\bar{B} \bar{F} \bar{H}-\bar{A} \bar{G} \bar{H},
\end{aligned}
$$

via an algebraic computation. By the Routh-Hurwitz stability criterion, the roots are in $\mathbb{C}_{-}$if and only if $b_{3} b_{2}>b_{1}$ and $b_{3} b_{2} b_{1}>b_{1}^{2}+b_{3}^{2} b_{0}$.

We avoid the evaluation of the basic reproduction number $\Re_{\mathfrak{o}}$ for the two cases of age structured model using the next generation matrix [11] where $\mathfrak{R}_{\mathfrak{o}}$ is dependent on the parameters of the infectious disease, i.e., a real data set. We have already discussed the stability of infectious and epidemic states and the next generation matrix would determine a number, which is produced by transmission rates $\left(\beta_{A A}, \beta_{A B}, \beta_{C B}, \beta_{C C}\right)$. Those transmission rates are chosen according to the stability conditions and need not represent feasible values for a particular real disease. However, the scenario suits to the case of bacterial meningitis which could be transmitted by traveller adults to the children [9].

\section{Simulations for the model with age structure}

The simulations refer to a population consisting of 100 adults and 50 children. We assume that the birth and death rate are equal, $B_{A}=B_{A}=\mu=0.0128$. It seems not realistic, but we consider a small group of adults who have already visited a country on meningitis belt or have been for annual pilgrimage to Mecca. So, we aim to focus on the transmission rates in a small closed group and we do not expect an extreme change of the population density in a short period of time. Since we do not have any particular data set of bacterial meningitis, we choose $\beta_{A A}=0.2821$ as referred in the previous model. It is reported that the incidence of bacterial meningitis is 6.1 per 1000 among children and infants in Africa and South Asia, while the prevalence of the disease is highly dependent on the serotype of meningitis [1]. Thus, we assume to have a transmission rate among children $\beta_{C C}=0.61$ in case the children could not transmit the disease to adults, i.e. $\beta_{C A}=0$. Recovery rate remains as in adults model $r=0.6$ by taking into account that the recovery period is approximately 6 days [14]. We vary the infection rate between adults and children $\beta_{A C}$ to manipulate an epidemic or disease-free state of the population. We solve the system of differential equations numerically using the Matlab function 'ode45' and plot each scenario [21]. The stability state can be found in Table 5 for the endemic state.

Table 5. Stability of the disease-free state

\begin{tabular}{|c|c|c|c|c|c|c|c|}
\hline$B_{A}=B_{C}=\mu$ & $\beta_{A A}$ & $\beta_{A C}$ & $\beta_{C A}$ & $\beta_{C C}$ & $r$ & Stability of E1 & Stability of E2 \\
\hline 0.0128 & 0.2821 & $\mathbf{0 . 2}$ & 0 & 0.61 & 0.6 & stable & stable \\
0.0128 & 0.2821 & $\mathbf{0 . 0 2}$ & 0 & 0.61 & 0.6 & stable & stable \\
0.0128 & 0.2821 & $\mathbf{0 . 0 0 2}$ & 0 & 0.61 & 0.6 & stable & unstable \\
0.0128 & $\mathbf{0 . 3}$ & 0.2 & 0 & 0.61 & 0.6 & stable & stable \\
0.0128 & $\mathbf{0 . 0 2}$ & 0.2 & 0 & 0.61 & 0.6 & stable & stable \\
0.0128 & $\mathbf{0 . 0 1}$ & 0.2 & 0 & 0.61 & 0.6 & unstable & unstable \\
\hline
\end{tabular}

The presented model points out the importance of the variation of infection rate among two age classes. Choosing $\beta_{C A}=0$ not only simplifies the mathematical work but also makes sense for the infections diseases transported mainly by adults. For example, HIV 
infection is mostly carried by adults and treats the children using adults as a reservoir. This model aims to explain the mechanism of such an infection which is carried by adults to children using some published information about bacterial meningitis. Figure 4 shows the simulation result for the case $I_{A}(0)=1$ and $I_{C}(0)=1$ for different transmission rates.

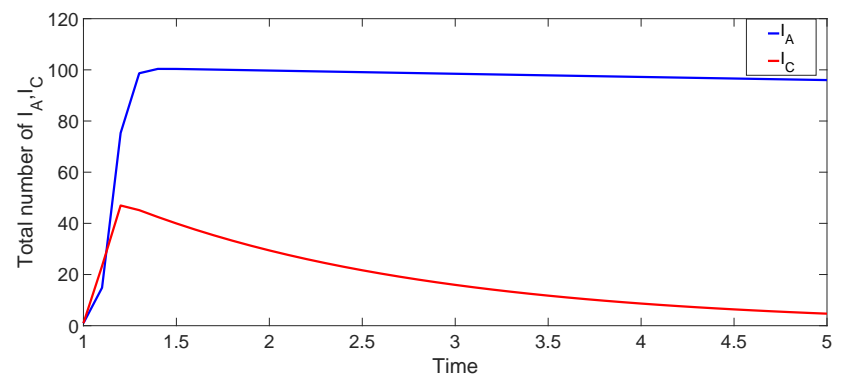

(a) $\beta_{A A}=0.2821$ and $\beta_{A C}=0.2$

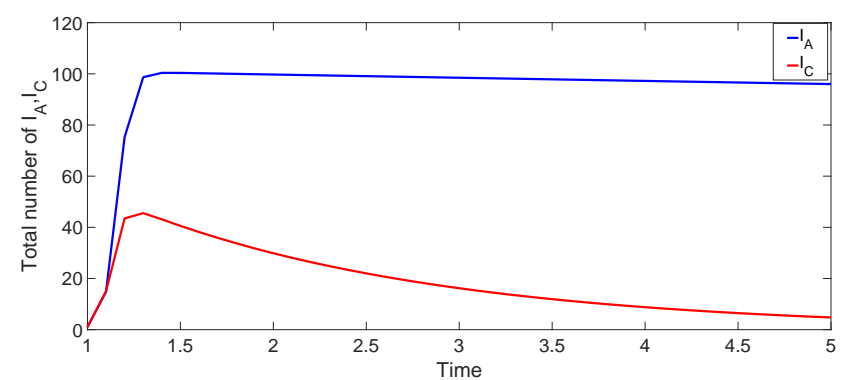

(b) $\beta_{A A}=0.2821$ and $\beta_{A C}=0.002$

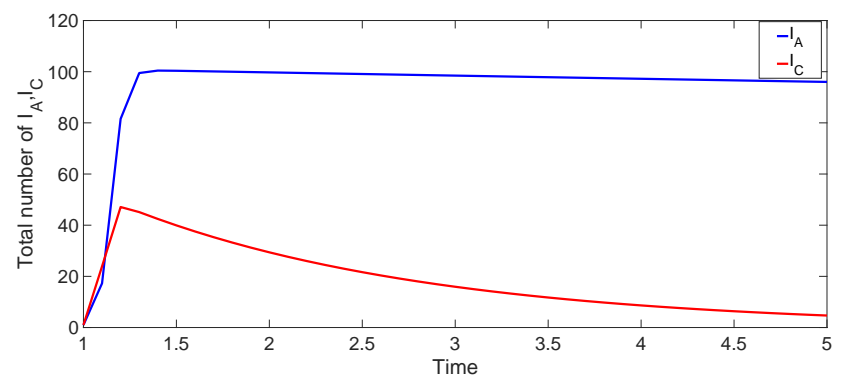

(c) $\beta_{A A}=0.3$ and $\beta_{A C}=0.2$

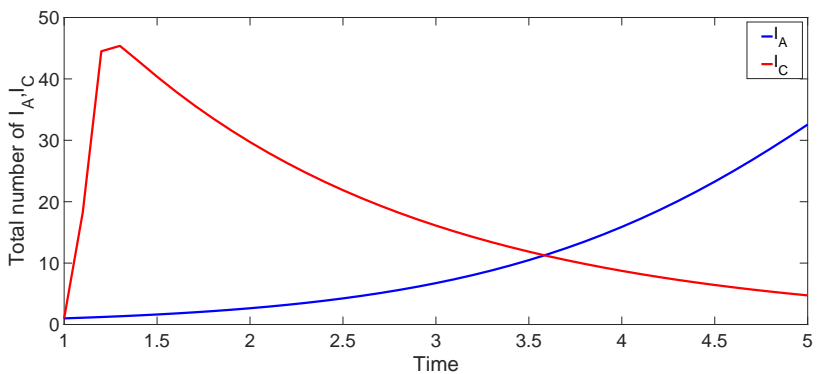

(d) $\beta_{A A}=0.01$ and $\beta_{A C}=0.2$

Figure 4. The variation of the $I_{A}$ and $I_{C}$ classes for various values of the infectious rate $\beta_{A A}$ and $\beta_{A C}$. (a) $\beta_{A A}=0.2821$ and $\beta_{A C}=0.2$, (b) $\beta_{A A}=0.2821$ and $\beta_{A C}=0.002$, (c) $\beta_{A A}=0.3$ and $\beta_{A C}=0.2$, (d) $\beta_{A A}=0.01$ and $\beta_{A C}=0.2$ 


\section{Conclusion}

We have presented two mathematical models for a infection scenario where the traveller adults transmit a disease to their environment. In the first model we analysed how the transmission rate of disease between adults effects the stability of disease-free and endemic case. In reality, there are many infectious diseases (HIV, Hepatitis B, Palio, meningitis etc.) that include aspects of both models in their transmission mechanisms. In this sense we believe that the estimation of the transmission rate $\beta$ of the first model (2.1) would enlighten the estimation of the transmission parameter $\beta_{A C}$ of the second model (4.1). Although the transmission parameter $\beta_{A C}$ is very important for the spread of the disease, it is not always possible to obtain this value from a real data set. The simulations show the transmission behaviour of a disease -particularly bacterial meningitis- among adults and among adults and children, receptively. We have mainly concentrated on the mathematical interpretations of our model but the model is available to test for similar infection scenarios.

\section{References}

[1] S. Agrawal and S. Nadel, Acute bacterial meningitis in infants and children epidemiology and management, Pediatr Drugs, 13 (6), 385-400, 2011.

[2] O.M. Akpa and B.A. Oyejola, Modeling the transmission dynamics of HIV/AIDS epidemics: an introduction and a review, J. Infect. Dev. Ctries. 4 (10), 597-608, 2010.

[3] B.M. Althouse and S.V. Scarpino, Asymptomatic transmission and the resurgence of Bordetella pertussis, BMC Medicine, 13, 146, 2015.

[4] E.J. Anderson and S.G. Weber, Rotavirus infection in adults, Lancet Infect. Dis. 4, 91-99, 2004.

[5] R.M. Anderson and R.M. May, Infectious Diseases of Humans, Dynamics and Control, Oxford University Press, Oxford, 1991.

[6] P. Balmer, C. Burman, L. Serra and L. J. York, Impact of meningococcal vaccination on carriage and disease transmission: A review of the literature, Hum. Vaccin. Immunother, 14 (5), 1118-1130, 2018.

[7] S. Bunimovich-Mendrazitsky and L. Stone, Modeling polio as a disease of development, J. Theor. Biol. 237 (3), 302-315, 2005.

[8] Centers for Disease Control and Prevention, https://www.cdc.gov/meningitis/bacterial.html

[9] M. Ceyhan, M. Celik, E.T. Demir, V. Gurbuz, A.E. Aycan and S. Unal Acquisition of meningococcal serogroup $W-135$ carriage in turkish hajj pilgrims who had received the quadrivalent meningococcal polysaccharide vaccine, Clin. Vaccine Immunol. 20 (1), 66-68, 2012.

[10] S. Chávez-Bueno and, G.H. Jr. McCracken, Bacterial meningitis in children, Pediatr Clin. N. Am. 52, 795-810, 2005.

[11] P. van den Driessche and J. Watmough, Reproduction numbers and sub-threshold endemic equilibria for compartmental models of disease transmission, Math. Biosci. 180, 29-48, 2002.

[12] D.J.D. Earn, P. Rohani, B.M. Bolker and B.T. Grenfell, A simple model for complex dynamical transitions in epidemics, Science, 87, 667-670, 2000.

[13] T. Harko, S.N.F. Lobo and M.K. Mak, Exact analytical solutions of the SusceptibleInfected-Recovered (SIR) epidemic model and of the SIR model with equal death and birth rates, Appl. Math. Comput. 236, 184-194, 2014.

[14] O. Hoffman and R.J. Weber, Pathophysiology and treatment of bacterial meningitis, Ther. Adv. Neurol. Disord. 2(6), 1-7, 2009.

[15] T.J. Irving, K.B. Bltuss, C. Colijn and C. L. Trotter, Modelling meningococcal meningitis in the African meningitis belt, Epidemiol. Infect. 140 (05), 89-905, 2011. 
[16] D.S. Jones and B.D. Sleeman, Differential Equations and Mathematical Biology, Chapman and Hall/CRC, London, 2003.

[17] D. Kalajdzievska and M.Y. Li, Modeling the effects of carriers on transmission dynamics of infectious diseases, Math. Biosci. Eng. 8, 3, 2011.

[18] W.O. Kermack and A.G. McKendrick, Contribution to mathematical theory of epidemics, Soc. Lond. A Mat. 115, 700-721, 1927.

[19] Y.A. Kuznetsov and C. Piccardi, Bifurcation analysis of periodic SEIR and SIR epidemic models, J. Math. Biol. 32, 109-121, 1994.

[20] C. Ma, S. Yan, Q. Su, L. Hao, S. Tang, Z. An, Y. He, G. Fan, L. Rodewald and H. Wang, Measles transmission among adults with spread to children during an outbreak: Implications for measles elimination in China, 2014, Vaccine, 34 (51), 6539-6544, 2016.

[21] MATLAB, version 9.14.0.813654 (R2018a), The MathWorks Inc., Massachusetts, 2018.

[22] A.M. Molesworth, L.E. Cuevas, S.J. Connor, A.P. Morse and M.C. Thomson, Environmental risk and meningitis epidemics in Africa, Emerg. Infect. Dis. 9 (10), 12871293,2003

[23] J. Müller and C. Kuttler, Methods and Models in Mathematical Biology, SpringerVerlag, Berlin, Heidelberg, 2015.

[24] J.D. Murray, Mathematical Biology, Springer-Verlag, New York, 1993.

[25] L.F. Olsen and W.M. Schaffer, Chaos versus noisy periodicity: alternative hypotheses for childhood epidemics, Science, 249, 499-504, 1990.

[26] A.M. Oordt-Speets, R. Bolijn, R.C. van Hoorn, A. Bhavsar and M. Kyaw, Global etiology of bacterial meningitis: A systematic review and meta-analysis, PLoS One, 13 (6), e0198772, 2018.

[27] Y. Özsürekci, Turkiye'de menenjite neden olan bakteriel ajanlar ve meningokal serogruplarin seroprevelansi (Unpublished doctoral dissertation), Hacettepe University Faculty of Medicine, 2013.

[28] K. Rock, S. Brand, J. Moir and M.J. Keeling, Dynamics of infectious diseases, Rep. Prog. Phys. 77, 026602, 2014.

[29] D. Schenzle, An age-structured model of pre-and post-vaccination measles transmission, Math. Med. Biol. 1, 169-191, 1984.

[30] R. Tekin, E.C. Dinleyici, M. Ceyhan, A. Karbuz, N. Salman, M. Sutcu, Z.Kurugol, Y. Balliel, M.Celik, M. Hacimustafaoglu, N. Kuyucu, M.Kondolot, G. Sensoy, O.Metin, S.S. Kara, M. Dinleyici, O.Kilic, C. Bayhan, V. Gurbuz, E. Aycan, A. Memedova, A. Karli and S. Celebi, The prevalence, serogroup distribution and risk factors of meningococcal carriage in adolescents and young adults in Turkey, Hum. Vaccin. Immunother. 13(5), 1182-1189, 2017. 